\title{
EEG Revealed That Fragrances Positively Affect Menopausal Symptoms in Mid-life Women
}

\author{
Sun Ae Moon ${ }^{1}$, Jisub Bae ${ }^{1}$, Kwangsu Kim ${ }^{1}$, Si Young Cho², Gusang Kwon ${ }^{2}$, Ran Lee ${ }^{2}$, \\ Seung Ho $\mathrm{Ko}^{2}$, Soyeon $\mathrm{Lim}^{2}$ and Cheil Moon ${ }^{1,3,4 *}$ \\ ${ }^{I}$ Department of Brain and Cognitive Sciences, Graduate School, Daegu Gyeungbuk Institute of Science and Technology, \\ Daegu 42988, ${ }^{2}$ AMOREPACIFIC R\&D Center, Yongin 17074, ${ }^{3}$ Convergence Research Advanced Centre for Olfaction, \\ Daegu Gyeungbuk Institute of Science and Technology, Daegu 42988, ${ }^{4}$ Convergence Brain Research Department, \\ Korea Brain Research Institute (KBRI), Daegu 41062, Korea
}

\begin{abstract}
During mid-life, women experienced not only physical but also neurological transition. Because of this, many women suffer from physiological and/or psychological menopausal symptoms. Although hormone therapy (HT) was broadly used to alleviate menopausal symptoms, HT showed inconsistent effects in case of psychological symptoms. Moreover, mid-life women's brains have distinct characteristics than in other periods of life, it is needed to study psychological symptoms in shifted brain network of mid-life women. As an alternative, inhalation of fragrances may alleviate psychological menopausal symptoms. To characterize the alleviation mechanism by fragrances, we tested the effect of fragrances on menopausal symptoms using electroencephalographic (EEG) methods. We hypothesized that fragrance could restore mid-life women's brain response to stressful situations. We tested six fragrance conditions, including no-odor condition (solvent only) in twenty-eight mid-life women (49.75 years \pm 3.49 ). Our results showed that fragrances increased alpha power and decreased $\beta / \alpha$ ratio depending on the severity of menopausal symptoms in a stressful situation. Our study would be helpful in psychological menopausal symptom alleviation as well as fragrance screening for well-being in mid-life.
\end{abstract}

Key words: Fragrance, Middle aged, Female, EEG, Depressive symptoms, Anxiety

\section{INTRODUCTION}

Many mid-life women suffer from physiological and/or psychological menopausal symptoms [1]. They experience a menopausal transition period called 'perimenopause,' which is defined as the period immediately before menopause and the first year after menopause [2]. Although cessation of menstruation is unavoidable, women who have severe menopausal symptoms have a very low quality of life ratings [3]. Women may experience a more negative mood during perimenopause than in other periods of life,

Submitted August 18, 2020, Revised September 18, 2020,

Accepted September 28, 2020

* To whom correspondence should be addressed. TEL: 82-53-785-1040, FAX: 82-53-785-6109 e-mail:cmoon@dgist.ac.kr which may lead to mood disorders [4-6].

Mid-life women's brains have distinct characteristics than in other periods of life. During mid-life, women experienced not only physical but also neurological transition [1]. According to Frey et al. [7], the brain network for emotional regulation may shift during the menopausal transition and early postmenopausal years. They suggested that these changes possibly contribute to the occurrence of mood and anxiety symptoms in midlife women (perimenopausal/postmenopausal women). Midlife women displayed increased activation in the dorsolateral prefrontal cortex during the emotional task, although amygdala engagement was not revealed. Amygdala showed significant deactivation in response to fearful faces. This brain activity was contrary to the observation in young adults [8]. Another study demonstrated significantly different EEG mapping between the menopausal syndrome patients with depression and healthy controls [9]. Their results suggest that 
a deteriorated vigilance in the mid-life women's brain could lead to a decreased ability to adapt the daily problems in a complicated life.

Although attempts have been made to alleviate psychological menopausal symptoms, most studies approached from a hormone perspective. Estrogen-containing hormone therapy (HT) was used to treat menopausal symptoms, however, compared to the primary indication of HT, such as vasomotor symptoms, the reported alleviation effects in the neurological functions were inconsistent [10]. In addition, the functional magnetic resonance imaging study revealed that HT does not modify emotion-induced brain activity in postmenopausal women [11]. The brain activity of the amygdala or ventrolateral prefrontal cortex showed no significant difference between the HT group and the control group when presenting negative, neutral, and positive scenes. They concluded that HT did not affect recognition, valence, or arousing ratings in older women. Furthermore, HT usage in mid-life women has decreased since the Women's Health Initiative study in 2002 [12]. This study was stopped early because of increased risks of breast cancer, pulmonary embolism, coronary heart disease, and stroke than the placebo group. And it affected concerns towards HT use among mid-life women [13].

As an alternative, inhalation of fragrances may alleviate psychological menopausal symptoms. Hedonic perception of fragrances modulates the physiological and psychological states of people [14]. Fragrances also are known to evoke various affective responses $[15,16]$. Pleasant fragrances tend to evoke positive moods, while unpleasant odors enhance negative moods [17-19]. Fragrances have reported stress-relief effects, as revealed using psychophysiological methods such as electromyography [20] and EEG [21]. Among all senses, olfaction closely interacts with emotion processing [22-24]. The olfactory neuroanatomy shows monosynaptic axonal connections from the olfactory bulb to the primary emotion area, the amygdala $[25,26]$. This anatomical point of view explains that fragrances could interact with emotion processing in the brain [24]. In perimenopause, some fragrances reported alleviation effects in stress, depressive symptoms, and anxiety symptoms [19, 27-30]. Schiffman et al. [19] reported that participants exposed to pleasant fragrances for five days answered alleviation of their feelings of tension, depression, and confusion. Another study reported positive mood change and alleviation of menopausal symptoms after massage with essential oils [30]. These studies showed that fragrances could positively modulate the physiological and psychological states. In addition, fragrances showed alleviation of menopausal symptoms. However, it is not yet known how fragrances change brain signals in women suffering from menopausal symptoms.
It is important to study whether the fragrance acts on the brain of mid-life women to relieve psychological symptoms. Because we can understand how fragrances influence on the mid-life women, and characterize how fragrances change the shifted emotional brain network of mid-life women. Moreover, we can quantify fragrance effects directly with brain signals using EEG. Six EEG indexes were used to quantify fragrance effects: theta, alpha, beta, gamma [31], alpha asymmetry (valence index), and $\beta / \alpha$ ratio (arousal index) [32]. We observed correlation pattern changes between EEG indexes and psychological symptoms by fragrances. We also compared which fragrance could revert to brain signals as similar as before stress stimulation brain state using linear regression pattern changes.

In this study, we evaluated the effect of fragrances on mid-life women. We hypothesized that fragrance could restore mid-life women's brain response to the stressful situations compared to no odor condition. We presented negative affective pictures (NAPS), which can induce stressful valence [33]. For the fragrances stimuli, lavender $(\mathrm{L})$, olibanum $(\mathrm{O})$, palmarosa $(\mathrm{P})$, blended fragrances based on olibanum (O29), and blended fragrances based on palmarosa (P12) were mainly tested in this study. The 'lavender' was selected as a positive control because lavender has already been well studied its anxiolytic, sedative, anti-depressive, and calming properties through previous studies [34-36]. Other essential oils were selected by the pre-test. We measured EEG to observe emotional state changes in the brain and how influenced by fragrances. We focused on how fragrances changed EEG indexes, depending on menopausal symptom severity (Kupperman menopausal index (KI) [37]). This study will be helpful in understanding how midlife women maintain their mental health despite the increasing risks of mood disorders.

\section{MATERIALS AND METHODS}

\section{Participants}

Thirty mid-life women participated in this study; one of them was excluded because of task non-compliance and another one because of health problems ( $\mathrm{n}=28$, mean age: $49.75 \pm 3.49$, age range: 43 56) (S1 Table). All participants were right-handed and had no history of psychological or neurologic diseases. The olfactory function was tested by a Sniffin' Sticks test kit (Burghardt, Wedel, Germany), which determines odor threshold and discrimination ability [38]. These odor threshold tests and discrimination tests were performed shortly before recording EEG. Only those who passed the odor threshold and discrimination ability could participate in the experiment. The experimental procedures were approved by the Clinical Research Information Service (KCT0005318), and the 
Institutional Review Board Ethics Committee, Daegu Gyeongbuk Institute of Science and Technology (DGIST-160909-HR-015-02). All participants gave their written informed consent prior to participating in the experiment. All experiments were performed in accordance with all relevant guidelines and regulations.

\section{Negative affective pictures preparation}

Negative affective pictures were prepared using the database of the Nencki Affective Picture System (NAPS) [33], which contains 1,356 high-quality pictures of five categories: people, faces, animals, objects, and landscapes. To induce negative affective emotion during the experiment, we selected the high arousal, and low valence scored pictures by women participants to make visual stimuli (S2 Table). The score range was from 1 to 9 , which represented 1 means negative valence and low arousal. We prepared six sets of visual stimuli (20 negative valence pictures per set; 500-ms blank between pictures) to avoid presenting repeated stimulifive categories of pictures evenly distributed for these six sets. The score of each set is significantly different from the middle (i.e., score 5). There was no significant difference between each NAPS set (Arousal; 6.10 \pm 0.09 (SD), Valence; $3.54 \pm 0.05$ (SD)).

\section{Fragrance preparation}

Lavender $(\mathrm{L})$, olibanum $(\mathrm{O})$, palmarosa $(\mathrm{P})$, blended fragrances based on olibanum (O29), and blended fragrances based on pal- marosa (P12) were mainly tested in this study ('lavender, Lavandula angustifolia oil,' 'olibanum, Boswellia carterii oil,' 'palmarosa, Cymbopogon martini oil from Robertet, Grasse, France). These fragrances were given from Amore Pacific (Yong-In, Republic of Korea), and selected by preference test (S1 Fig.). Blended fragrances O29 and P12 were made based on the olibanum and palmarosa each (also given by Amore Pacific). O29, which contains mainly olibanum essential oil, also contains lavender, palmarosa, patchouli, chamomile, clary sage, shiu, and nutmeg. P12, contains mainly palmarosa, also contains lavender, patchouli, bergamot, rose, shiu and ylangylang. The fragrances of essential oils were diluted to $3 \%(\mathrm{v} / \mathrm{v})$ in dipropylene glycol. Each diluted fragrance $(20 \mu \mathrm{l})$ was dropped on filter paper, which was then placed in a conical tube until presented to participants. Each tube was labeled with a number for a blind test. For the blind test, each filter paper was presented about $2 \mathrm{~cm}$ below the nostrils by hand during the fragrance conditioning period for $10 \mathrm{~s}$ (Fig. 1).

\section{Experimental procedure}

EEG and skin conductance were recorded in an olfactory chamber. Participants had time to stabilize their mood for about $1 \sim 2$ min before the trial began. Participants sat in front of a monitor screen; the following presentation order was used: baseline period (10s) - negative affective pictures presentation (90s) - fragrance period (10 s) (Fig. 1). During fragrance period, the participants

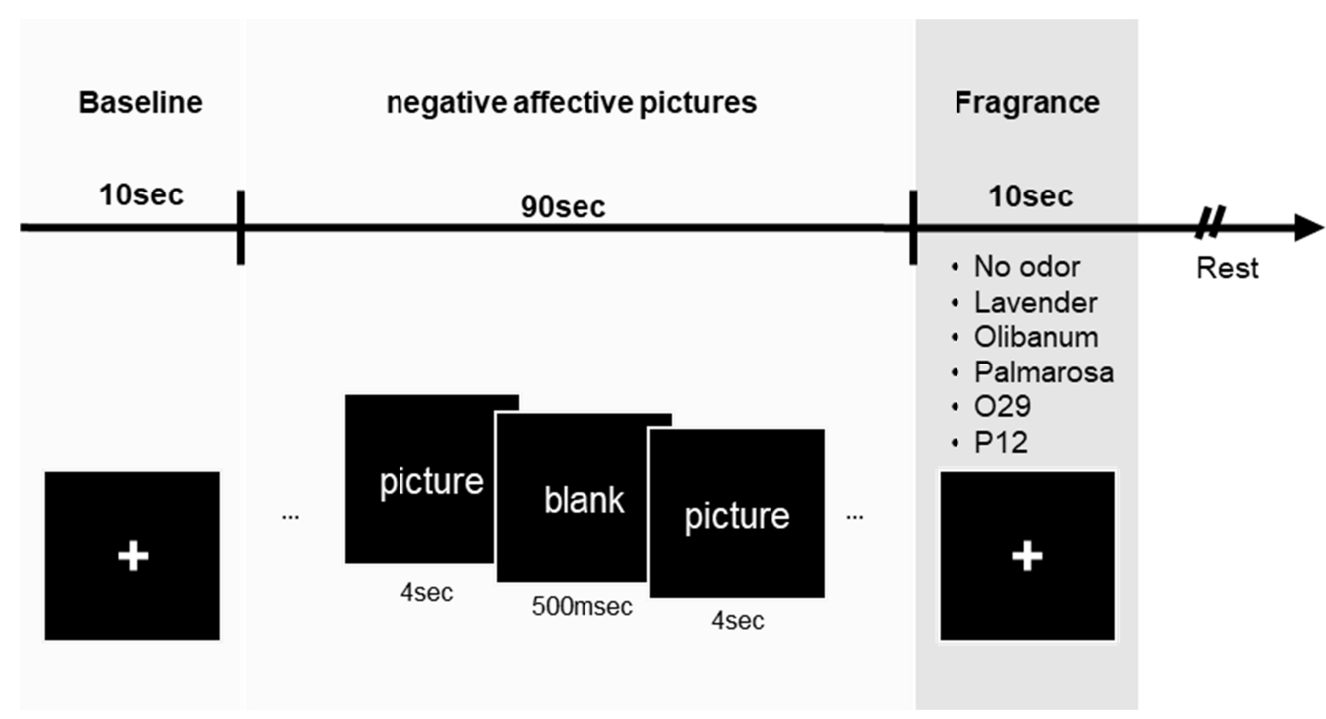

Fig. 1. Experimental scheme. EEG recording followed the following order: baseline period ( $10 \mathrm{~s})$ - negative affective stimulus ( $90 \mathrm{~s})$ - fragrance period (10 s) - rest. During EEG recording, a monitor screen was presented to the participants (black squares). In the baseline period, participants' eyes were fixed on a crosshair on the screen. Negative affective stimuli (low valence and high arousal) were then presented. A set consisted of 20 Nencki Affective Picture System pictures (4 s/image). Between pictures, a blank was presented for $500 \mathrm{~ms}$. During odor conditioning, the participant smelled fragrance (O29, P12, lavender, olibanum, or palmarosa) or odorless solution (3\% solvent). The fragrance presentation was randomly ordered. Each set followed the same process. Six different odor conditions were measured per subject. For the analysis, 'fragrance' and 'baseline' periods were used. O29: olibanum-based blended oil; P12: palmarosa-based blended oil. 
inhalated randomly presented with one of the fragrances or odorless solvent (no-odor condition). After each fragrance test set was completed, participants responded on a 7 -point scale as to how fragrance relieved tension in the previous set of experiments. Then, participants took a rest during olfactory chamber ventilation for at least 1 minute. Each participant attended EEG and skin conductance recording for all conditions in one day.

\section{Survey}

The severity of participants' menopausal symptoms was determined using the KI [37]. The total KI score was calculated according to a previous study. Factors had different weighting during the calculation: hot-flash score (X4), sweating (X2), sleep disorders (X2), irritability (X2), depressed mood (X1), dizziness (X1), tiredness (X1), joint pain (X1), headaches (X1), heart pounding or palpitations (X1), genital problems (X1). Menopausal symptoms were analyzed separately in five categories: 'depressive symptoms', 'anxiety symptoms,'somatic symptoms,' vasomotor symptoms', and 'vaginal dryness' [39]. Depressive symptoms contained three items: feeling unhappy or depressed, feeling tired or lacking energy, irritability. Anxiety symptoms were difficulty in sleeping and fast or strong heartbeat. Somatic symptoms contained three items: muscle and joint pains, headaches, and feeling dizzy or fainting. Vasomotor symptoms were hot flushes and night sweats. The vaginal dryness category included vaginal dryness and reduced secretion. The answers were rated as follows: none (0), mild (1), moderate (2), and severe (3). Scores of categories were averaged and compared with scores of other categories.

Fragrance relaxed effect was measured on 7-point scales after each test set, which showed NAPS. The question was written in Korean. Participants answered to 'The tension seems to be alleviating. after inhalation fragrances. The answers were rated as follows: strongly disagree (1), disagree (2), somewhat disagree (3), neutral (4), somewhat agree (5), agree (6), strongly agree (7). Participants answered to "How do you like this fragrance?" for rating fragrance preference. The answers were rated as follows: dislike extremely (1), dislike very much (2), dislike slightly (3), neither like nor dislike (4), like slightly (5), like very much (6), like extreamly (7).

\section{Autonomic sympathetic activity measure and analysis}

Skin conductance is measuring changes in sympathetic arousal, which can be quantified using two points of skin contact [40]. It is known as the only autonomic psychophysiological variable which is not contaminated by parasympathetic activity. To use as an index of sympathetic activity, we measured skin conductance using Biopac MP 36 system (sampling rate=1,000 Hz) (BIOPAC Systems., Goleta CA, USA). The raw data was obtained using Biopac
Student Lab software (BIOPAC Systems., Goleta CA, USA), and further analysis was done with MATLAB R2018a (MathWorks, Natick, MA, USA). The skin conductance level (SCL; reflect general changes in autonomic arousal) was calculated by (average SCL data during fragrance periods) - (average SCL data during baseline periods).

\section{EEG data acquisition}

EEG was recorded using an EEG amplifier (Active Two, Biosemi, Amsterdam, Netherlands) with an 8-channel system (sampling rate $=2,048 \mathrm{~Hz}$ ). The measured 8-channel sites were AF3, AF4, C1, $\mathrm{C} 2, \mathrm{C} 5, \mathrm{C} 6, \mathrm{O} 1$, and $\mathrm{O} 2$. The eight channels were located at the prefrontal cortex (AF3, AF4), pre-parietal cortex (C1, C2), and anterior and posterior transverse temporal cortex (C5, C6), and occipital cortex $(\mathrm{O} 1, \mathrm{O} 2)$. These locations corresponded to the left and right frontal, parietal, temporal, and occipital lobes. The Ag/ $\mathrm{AgCl}$ scalp electrodes were placed on an EEG cap (64ch, Biosemi), which was placed on channels followed by a 10 20 international system. We used the common-mode-sensor (CMS) and drivenright-leg (DRL) electrodes as reference electrodes. During recording, impedances were below kept $15 \mathrm{k} \Omega$.

\section{EEG pre-processing and power spectrum analysis}

For data extraction, the sampling rate of 2,048 Hz was reduced to $512 \mathrm{~Hz}$. The 8 EEG channels were filtered offline with a band-pass between 0.5 and $100 \mathrm{~Hz}$ to exclude noise caused by skin potentials and muscle artifacts. For data analysis, we extracted the power spectral density from the raw EEG data sets. We extracted four frequency bands: theta $(4 \sim 8 \mathrm{~Hz})$, alpha $(8 \sim 13 \mathrm{~Hz})$, beta $(13 \sim 30 \mathrm{~Hz})$, and gamma $(30 \sim 40 \mathrm{~Hz})$. Epoch for analysis was extracted from the baseline and fragrance conditioning periods. Each averaged power spectral density during the fragrance presentation was normalized by the baseline ('fragrance condition period' - 'baseline'). Alpha asymmetry was used as a valence index. Low alpha asymmetry was interpreted as negative emotions [41]. The frontal alpha power asymmetry index (AF4 - AF3) was calculated to measure negative feelings. $\beta / \alpha$ ratio was used as an arousal index. The beta wave is associated with the alert state of mind, whereas the alpha wave is higher in relaxed individuals [32]. The $\beta / \alpha$ ratio of each channel was calculated during odor conditioning. Then, the $\beta / \alpha$ ratio during the baseline period was subtracted from that during the fragrance conditioning periods for each channel for normalizing. Representative data were the mean values of the eight channels. The EEG was analyzed with MATLAB R2018a (MathWorks, Natick, MA, USA) and EEGLAB toolbox (EEGLAB, Boston, MA, USA) [42]. 


\section{Statistical analysis}

For statistical analysis, we used GraphPad Prism 5 software (GraphPad Software, Inc., La Jolla, CA, USA) and MATLAB R2018a. To assess the relationships between menopausal symptom severity and brain activity, we used Spearman's correlation. For each statistical analysis, we used a two-tailed p-value. Linear regression analysis was applied as indicators of pattern changes after presenting fragrances. The line of best-fit was calculated using GraphPad Prism 5 software. For detecting outliers, a cook's distance was used.

\section{RESULTS}

To understand the effects of fragrance on the negative emotion of mid-life women, we first set an experimental design that induces negative affective emotion. For inducing negative affective emotion, we made negative affective pictures (NAPS) set using the NAPS database [33] (Fig. 1). We made 6 set for inducing negative emotion (no-odor, L, O, P, O29, P12). Each set consisted of significantly low valence and high arousal pictures and was no significantly different among the set (S2 Table). We focused on changes
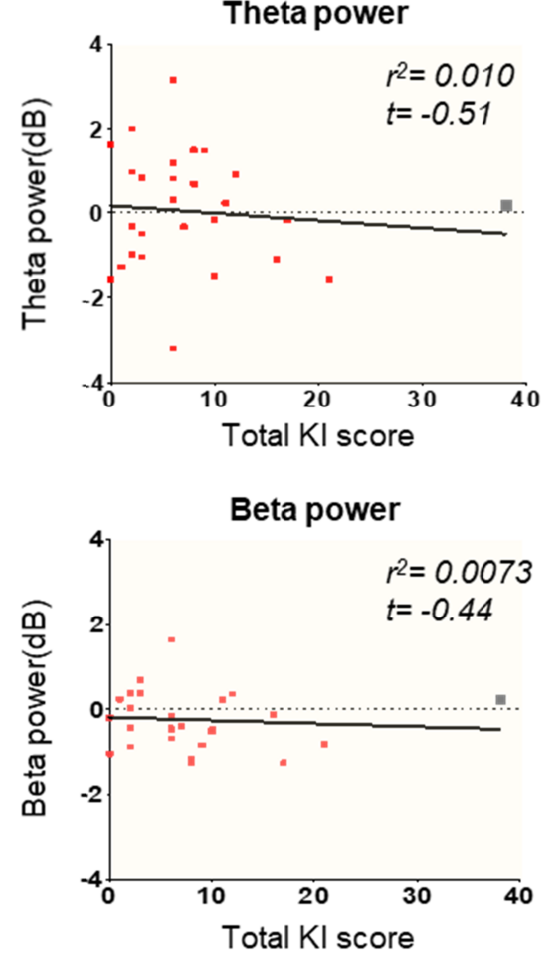

e

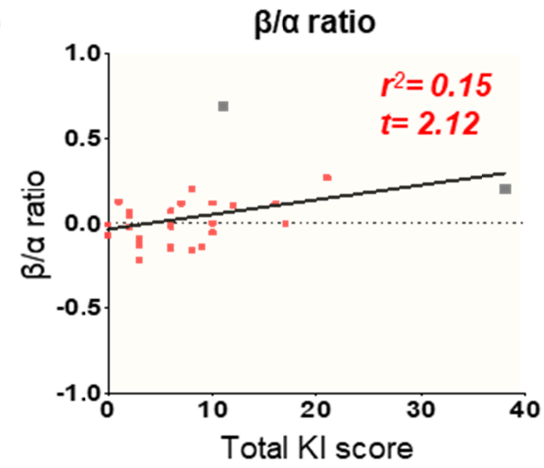

b

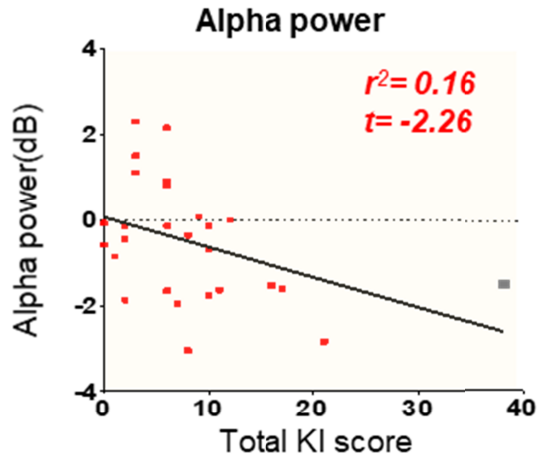

d

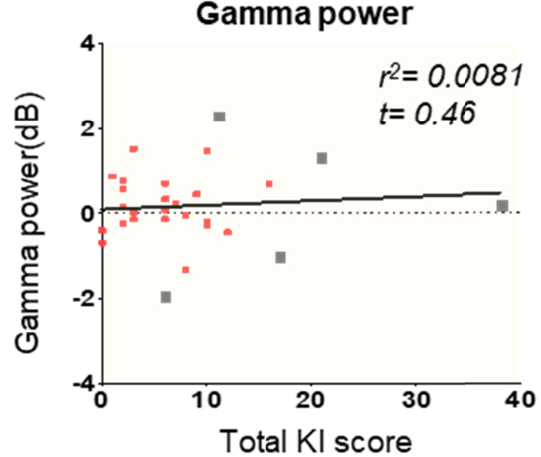

f

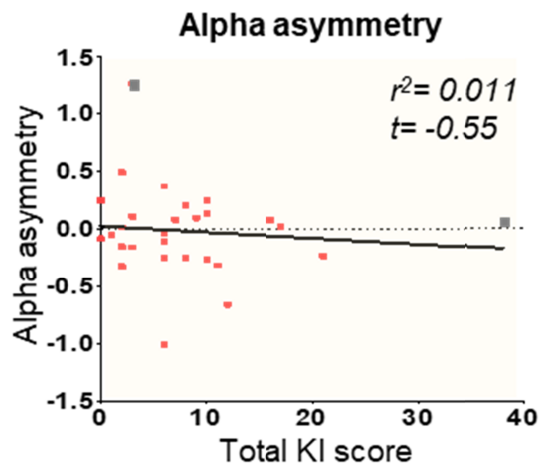

Fig. 2. Regression analysis of the four EEG frequency band powers, $\beta / \alpha$ ratio, and alpha asymmetry on the total KI score. Alpha power and $\beta / \alpha$ ratio showed a correlation with total KI score after negative affective stimuli in comparison with baseline brain activity. Red dots: individual data points; gray dots: outliers; black lines: linear regression patterns (Bonferroni-corrected values 0.0083 ) (a $\sim$ d). Regression pattern between EEG frequency band powers and total KI score under no-odor conditions. The EEG responses shown were normalized by subtracting the responses during the baseline period from those during odor conditioning. (e) Regression pattern between $\beta / \alpha$ ratio (arousal index) and total KI score. (f) Regression pattern between alpha asymmetry (valence index) and total KI score. Outliers represented as gray color squares (See excluded outliers results S3 Table). 
in EEG indexes after presenting NAPS.

We found that the total KI score showed a tendency of correlated pattern with the alpha power and $\beta / \alpha$ ratio by regression analysis (Fig. $2 \mathrm{~b}$ and $\mathrm{e}, \mathrm{r}^{2}=0.16, \mathrm{r}^{2}=0.15$ each). Because regression analysis is vulnerable to outliers, we verified the regression model with outlier rejected data (S3 Table). Specifically, alpha power showed a marginally significant decrease by the total KI score increase $(\mathrm{t}=-2.58, \mathrm{p}=0.016$ ), and the $\beta / \alpha$ ratio shown the opposite pattern $(t=2.065, p=0.050)$. We found that alpha power increased model performance while using outlier rejected data, and the $\beta / \alpha$ ratio does not change as well. Age has no significant correlation with both total KI score (S2 Fig.) and EEG indexes (S3 Fig.).

Secondly, we performed a correlation analysis between EEG indexes and KI categories. KI survey can be categorized by symptoms as psychological and physiological [39]. Interestingly, we found that only psychological symptoms correlated with EEG indexes, specifically, the alpha power, beta power, and $\beta / \alpha$ ratio (Table 1; psychological symptoms column). Alpha power showed significantly correlated with the anxiety symptoms and marginally significantly with the depressive symptoms. Beta power showed marginally significantly with the anxiety symptoms, and $\beta / \alpha$ ratio showed significantly correlated with depressive symptoms and marginally significantly with the anxiety symptoms. However, physiological symptoms had no significant correlations with the EEG indexes (Table 1; physiological symptoms column).

We observed different alpha power, beta power, and $\beta / \alpha$ ratio after NAPS depending on menopausal factors (KI score, depressive symptoms, and anxiety symptom severity). Based on these indexes, we evaluated fragrances' effects on psychological menopausal symptoms in the brain. We used fragrances which selected by 'relaxation effect' and 'preference' of responses from mid-life women in the pre-test (S1 Fig.). Before evaluating fragrances' effect on the brain, we first verified whether fragrances affected behavioral or autonomic sympathetic activity. We found that except $P$, most of the fragrances rated significantly or marginally $(\mathrm{P} 12, \mathrm{p}=0.065)$ above score 4 (the middle) in 'relaxed effect' (Fig. 3a). Although no statistical significance, skin conductance showed decreased patterns compared to no-odor condition overall fragrance conditions (Fig. 3b).

To evaluate fragrances' effect in the brain, we made the five deviation data sets between fragrance conditions and no-odor condition (L, O, P, O29, and P12). Using deviation data sets, we firstly observed average patterns of alpha power, beta power, and $\beta / \alpha$ ratio during fragrance inhalation (Fig. 4a $\sim$ c). However, these average patterns showed no significant difference from no-odor condition (Two-sample t-test, two-tail). Subsequently, we performed a regression analysis between the deviation data set and menopausal factors (KI score, depressive symptoms, and anxiety symptoms). Each fragrances linear regression pattern showed the line that best fits of data points using ordinary least-squares regression (Fig. $4 \mathrm{~d} \sim \mathrm{l}$ ).

We found significantly correlated patterns between alpha power and anxiety symptoms (Fig. 4f). Moreover, the $\beta / \alpha$ ratio also showed a significant correlation between depressive and anxiety symptoms (Fig. 4k and l). Specifically, O29 and P12 significantly induce increasing alpha power, especially on having higher anxiety symptom participants ( $\mathrm{t}$ value $=2.63$ and $2.15 \mathrm{each}$ ) and $\mathrm{L}$ in $\beta / \alpha$ ratio also increase alpha power unless having less significance $(\mathrm{p}=0.055, \mathrm{t}=2.01)$. Depressive symptoms only have an increasing tendency with O29 $(\mathrm{p}=0.055, \mathrm{t}=2.01)$ but no statistically significant with other fragrances (Fig. 4e and S4 Table). In the $\beta / \alpha$ ratio, O29, P12, and L have significantly affected fragrances (Fig. $4 \mathrm{k}$ and $\mathrm{l}$ ).

O29 decreased $\beta / \alpha$ ratio on overall menopausal factors (Fig. 4j 1 and S4 Table, marginally significant on KI score, $\mathrm{p}=0.06$ ). O and $\mathrm{P}$ also induced a decreasing tendency on depressive symptoms (Fig. 4k and S4 Table, $\mathrm{p}=0.073, \mathrm{p}=0.062$ each). Subsequently, we found that these fragrances affect more strongly to participants who rated comparatively higher menopausal factors rather than affected overall or lower rating participants (S4 Fig.). Fragrances

Table 1. Correlation between EEG indexes and KI categories

\begin{tabular}{|c|c|c|c|c|c|}
\hline & \multicolumn{2}{|c|}{ Psychological symptoms } & \multicolumn{3}{|c|}{ Physiological symptoms } \\
\hline & $\begin{array}{l}\text { Depressive } \\
\text { symptoms }\end{array}$ & $\begin{array}{c}\text { Anxiety } \\
\text { symptoms }\end{array}$ & $\begin{array}{c}\text { Somatic } \\
\text { symptoms }\end{array}$ & $\begin{array}{l}\text { Vasomotor } \\
\text { symptoms }\end{array}$ & $\begin{array}{l}\text { Virginal } \\
\text { dryness }\end{array}$ \\
\hline Theta power & $-0.25(\mathrm{p}=0.20)$ & $0.03(\mathrm{p}=0.86)$ & $-0.11(p=0.58)$ & $0.00(\mathrm{p}=0.99)$ & $-0.15(p=0.44)$ \\
\hline Alpha power & $-0.34(\mathrm{p}=0.076)$ & $-0.43^{*}(\mathrm{p}=0.022)$ & $-0.18(\mathrm{p}=0.35)$ & $-0.24(\mathrm{p}=0.22)$ & $-0.03(\mathrm{p}=0.88)$ \\
\hline Beta power & $-0.09(\mathrm{p}=0.65)$ & $-0.36(p=0.064)$ & $-0.29(\mathrm{p}=0.13)$ & $-0.22(\mathrm{p}=0.26)$ & $0.24(\mathrm{p}=0.23)$ \\
\hline Gamma power & $0.33(\mathrm{p}=0.083)$ & $-0.02(\mathrm{p}=0.93)$ & $0.10(\mathrm{p}=0.61)$ & $-0.20(\mathrm{p}=0.30)$ & $0.21(\mathrm{p}=0.28)$ \\
\hline$\beta /$ a ratio & $0.40^{*}(\mathrm{p}=0.033)$ & $0.36(\mathrm{p}=0.061)$ & $0.14(\mathrm{p}=0.47)$ & $0.25(\mathrm{p}=0.20)$ & $0.10(\mathrm{p}=0.61)$ \\
\hline Alpha asymmetry & $0.00(\mathrm{p}=0.99)$ & $-0.20(\mathrm{p}=0.32)$ & $-0.09(\mathrm{p}=0.66)$ & $-0.06(\mathrm{p}=0.75)$ & $0.18(\mathrm{p}=0.37)$ \\
\hline
\end{tabular}

Spearman's correlation coefficients (rho) (two-tailed; in parentheses) are listed. Asterisks denote statistical significance $\left({ }^{*} \mathrm{p}<0.05\right)$. Alpha and beta power showed a significant correlation with psychological but no physiological symptoms. The $\beta / \alpha$ ratio also showed significant rho only for psychological symptoms. 


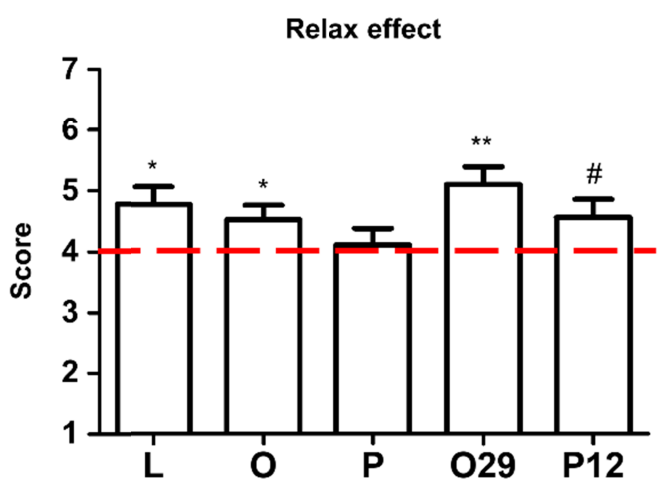

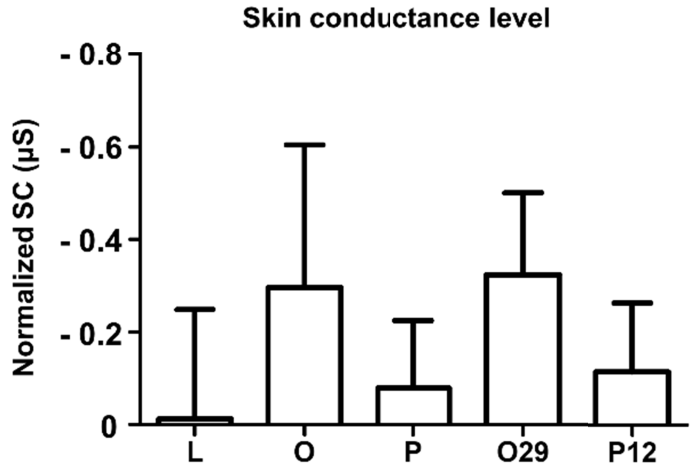

Fig. 3. Survey and physiological responses to fragrance after NAPS. (a) Survey results of the fragrance effect after NAPS. One sample t-test was performed to verify the difference from the middle (score 4, one-tail). (b) Skin conductance level of the fragrance effect after NAPS. Each SCL was calculated by (average SCL data during fragrance periods) - (average SCL data during baseline periods). Normalized skin conductance was calculated by each fragrance condition SCL- no odor condition SCL. Y-axis is inversed in this graph. Two sample t-test performed to verify the difference from no-odor condition (no-odor condition set to 0 , one-tail) $\left(\# \mathrm{p}<0.1,{ }^{*} \mathrm{p}<0.05,{ }^{* *} \mathrm{p}<0.01\right)$.

significantly decrease the slope of the no-odor regression patterns at $\beta / \alpha$ ratio but no significant changes in the $y$-axis direction. In contrast, no fragrance induced beta power change depending on menopausal factors.

We evaluated fragrances' effect, however, this may be very locally or dispersedly effect to the brain. To verify how fragrance globally affects the brain, we observed overall channels change by comparing no-odor and fragrance conditions (Fig. 5). We found that fragrances altered EEG indexes locally or dispersedly in total channels, especially alpha and beta, with the total KI and depressive symptoms (Fig. 5a, b, d, and e). In contrast, fragrances altered EEG indexes globally on anxiety symptoms compared to other menopausal factors (Fig. $5 \mathrm{c}$ and f). In the $\beta / \alpha$ ratio, most fragrances affected globally on overall menopausal factors (Fig. $5 \mathrm{~g} \sim \mathrm{i}$ ).

We additionally investigated whether the psychological evaluation of fragrance could reflect in the brain signal or not. We assumed that if fragrance preference has a positive correlation with alpha or negative correlation with $\beta / \alpha$ ratio, psychological influence may affect EEG in our experimental design. Thus, we performed the Spearman's correlation analysis between EEG indexes and the fragrance preference score (Fig. 6). We found that each fragrance preference scores showed a weak negative correlation with alpha power (number of XY Pairs $=140$, rho $=-0.22, \mathrm{p}=0.0078$, two-tailed), a weak positive correlation with beta power ( $\mathrm{rho}=0.18$, $\mathrm{p}=0.031$ ), and a weak positive correlation with $\beta / \alpha$ ratio $(\mathrm{rho}=0.24$, $\mathrm{p}=0.0041$ ).

\section{DISCUSSION}

Here, we found that fragrances increased alpha power and de- creased $\beta / \alpha$ ratio depending on the severity of menopausal symptoms in a stressful situation. To answer whether fragrance could positively modulate brain signals in mid-life women, we firstly verified brain signals (Fig. 2). From the previous studies, we could predict shifted brain network for emotional regulation [7], and menopausal symptom dependent EEG power [9]. We could characterize brain signals of midlife women in a stressful situation using negative affective pictures system (NAPS), which scored high arousal and negative valence [33]. We found psychological menopausal symptoms (not in physiological menopausal symptoms) correlated with EEG indexes, the alpha power, beta power, and $\beta / \alpha$ ratio (Table 1). Then, we observed whether fragrance could restore the symptom-dependent EEG indexes. Our EEG results verified the relaxing effects of fragrances [34, 36, 43-46]. The EEG pattern of Fragrances showed the possibility to alleviate menopausal symptoms [28, 30, 47, 48], reported previously, and showed similar relaxing effects with behavioral or autonomic sympathetic activity in Fig. 3. Interestingly, only EEG results showed that fragrances affect more strongly to mid-life women with severe menopausal symptoms (Fig. 4). The survey and skin conductance level showed no significant correlation with menopausal symptoms severity (S5 Fig.). By measuring brain signal, it was possible to reveal the effect of fragrance according to menopausal symptoms severity, which could not be verified by other measurement methods. The topographical EEG pattern showed that increased anxiety symptoms, EEG indexes from almost channels were more changed by fragrance, on the other hand, increased depressive symptoms, EEG indexes from locally or dispersedly within entire channels were more changed by fragrance (Fig. 5). It means that fragrances showed symptom-specific activation patterns in mid-life women's 
a

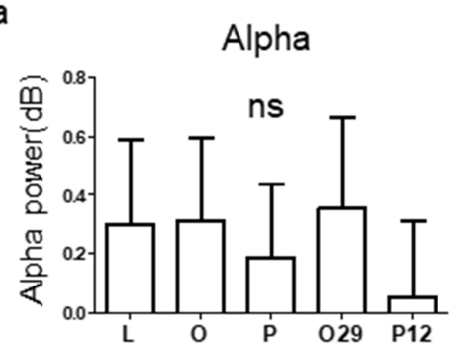

b

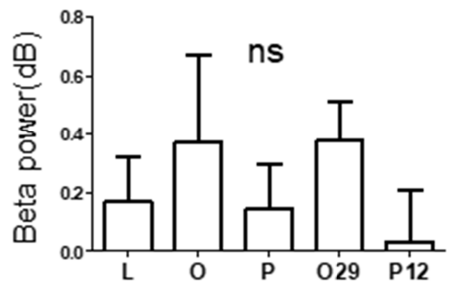

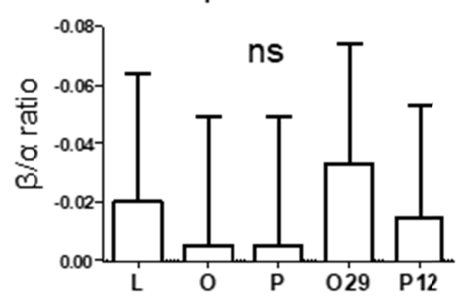

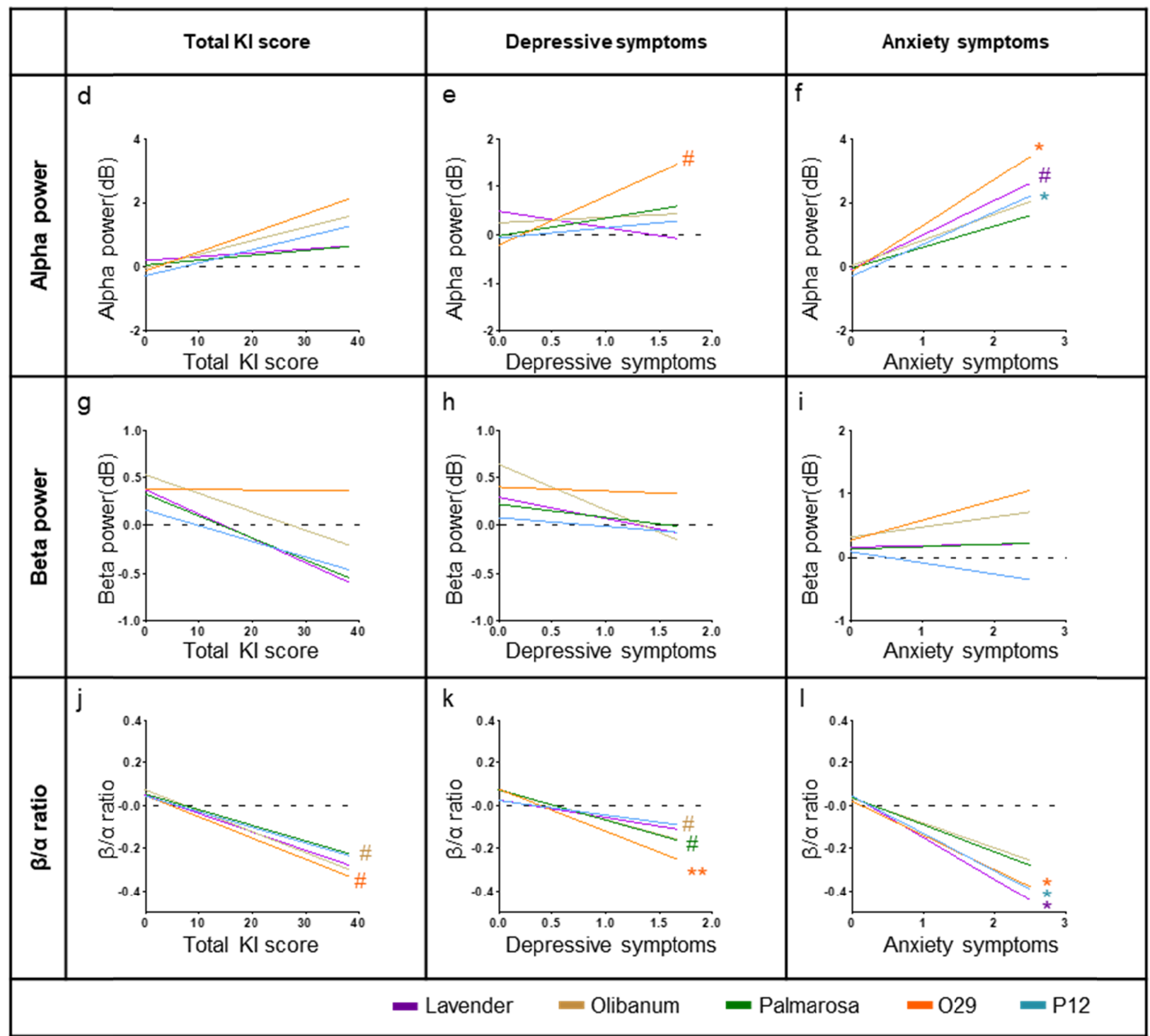

Fig. 4. EEG pattern changes while inhalation fragrances. (a $\sim$ c) EEG results of the fragrance effect after NAPS. Two sample t-test performed to verify the difference from no-odor condition (no-odor condition set to 0 , two-tail). (c) y-axis is inversed in this graph. (d l) Regression pattern changes during the inhalation of fragrances. Fragrances alter the slope of the linear regression of $(\mathrm{d} \sim \mathrm{f})$ alpha power, $(\mathrm{g} \sim \mathrm{i})$ alpha power, and $(\mathrm{j} \sim \mathrm{l}) \beta / \alpha$ ratio slope vs. total KI score, depressive symptoms, and anxiety symptoms. Black lines: no-odor condition (solvent). Color lines: odor conditions. All input data were deviation value between oil fragrance condition and no-odor condition data (e.g., O29 data - no-odor data). Asterisks denote statistical significance (\#p<0.1, $\left.{ }^{*} \mathrm{p}<0.05,{ }^{* *} \mathrm{p}<0.01\right)$. 


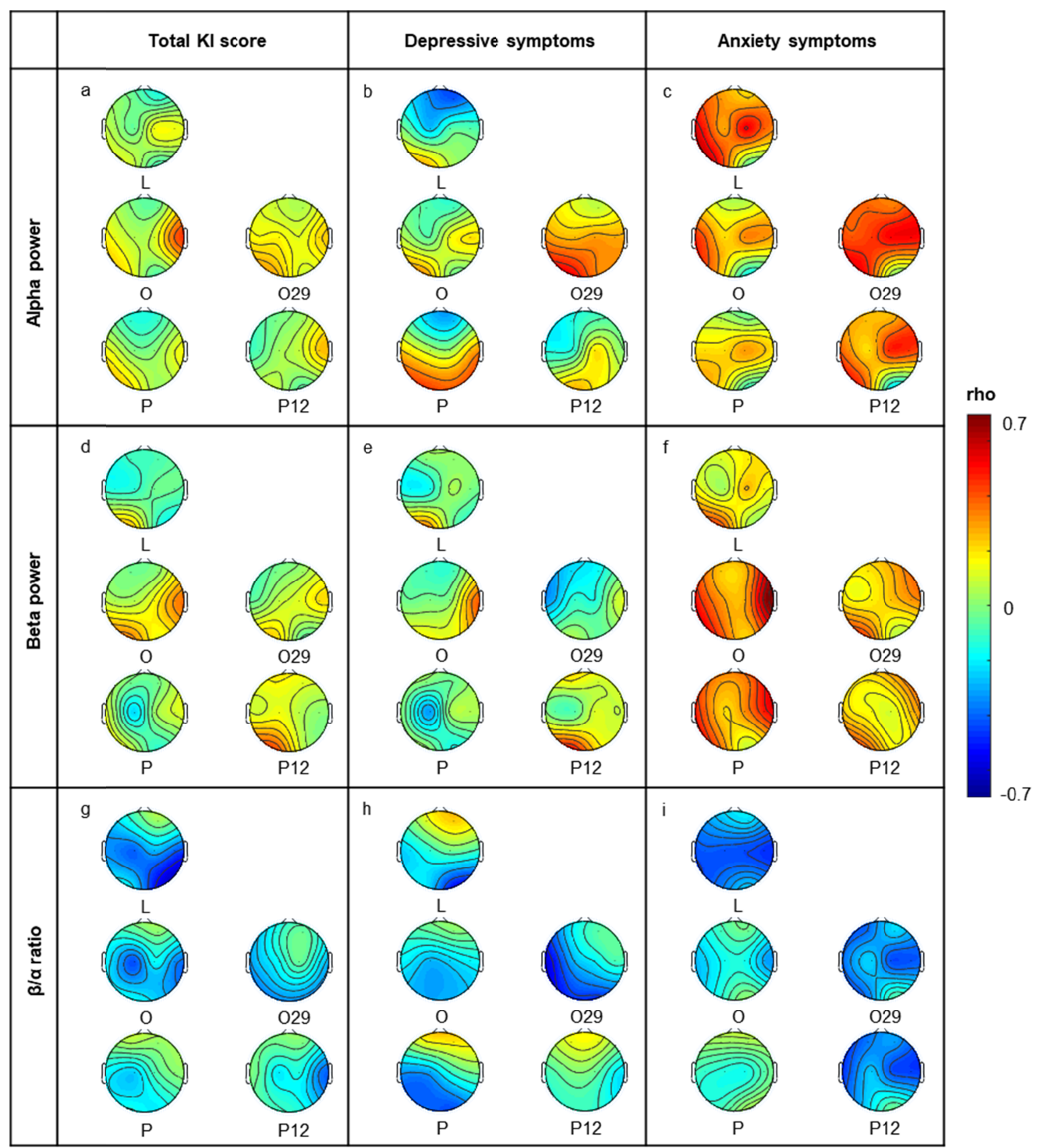

Fig. 5. Correlation patterns of total electrodes during inhalation fragrances. The topography represents changes of Spearman's rho [KI score \& EEG power] by fragrances. Presented topography data were normalized; Spearman's rho to show the correlation change by fragrances. The color of each channel represents the change range by each fragrance. Blue (increased KI score and decreased EEG power), Red (increased KI score, and increased EEG power). All input data were deviation value between oil fragrance condition and no-odor condition data.

brains. Lastly, we checked that psychological influence in our EEG data, and it showed a weak negative correlation with alpha and weak positive correlation with $\beta / \alpha$ ratio, which means that fragrance preference did not affect to modulation of relaxation effect related EEG indexes. Our results may suggest that fragrances could alleviate menopausal anxiety or depressive symptoms by modulating responses in the brain.

We found that alpha power and $\beta / \alpha$ ratio reflected the characteristics of brain response to NAPS according to psychological menopausal symptoms (Fig. 2, Table 1). In our study, by subdividing the KI questionnaires into psychological symptoms and physiological symptoms, we found that alpha power and $\beta / \alpha$ ratio were related to psychological but not physiological menopausal symptoms (Table 1). These alpha power and $\beta / \alpha$ ratio have reported the association with emotional changes [32, 49-51]; the state of relaxation or decreased anxiety corresponds to an increase in alpha activity [50]. Besides, alpha power shows a negative correlation with subjective arousal level ratings [49]. The $\beta / \alpha$ ratio is known as an index of the arousal state because beta power is associated with an alert or excited state, and alpha power is more dominant in a relaxed state [32, 51]. In this study, we could confirm the changes in alpha power and $\beta / \alpha$ ratio in the stressful conditions. 

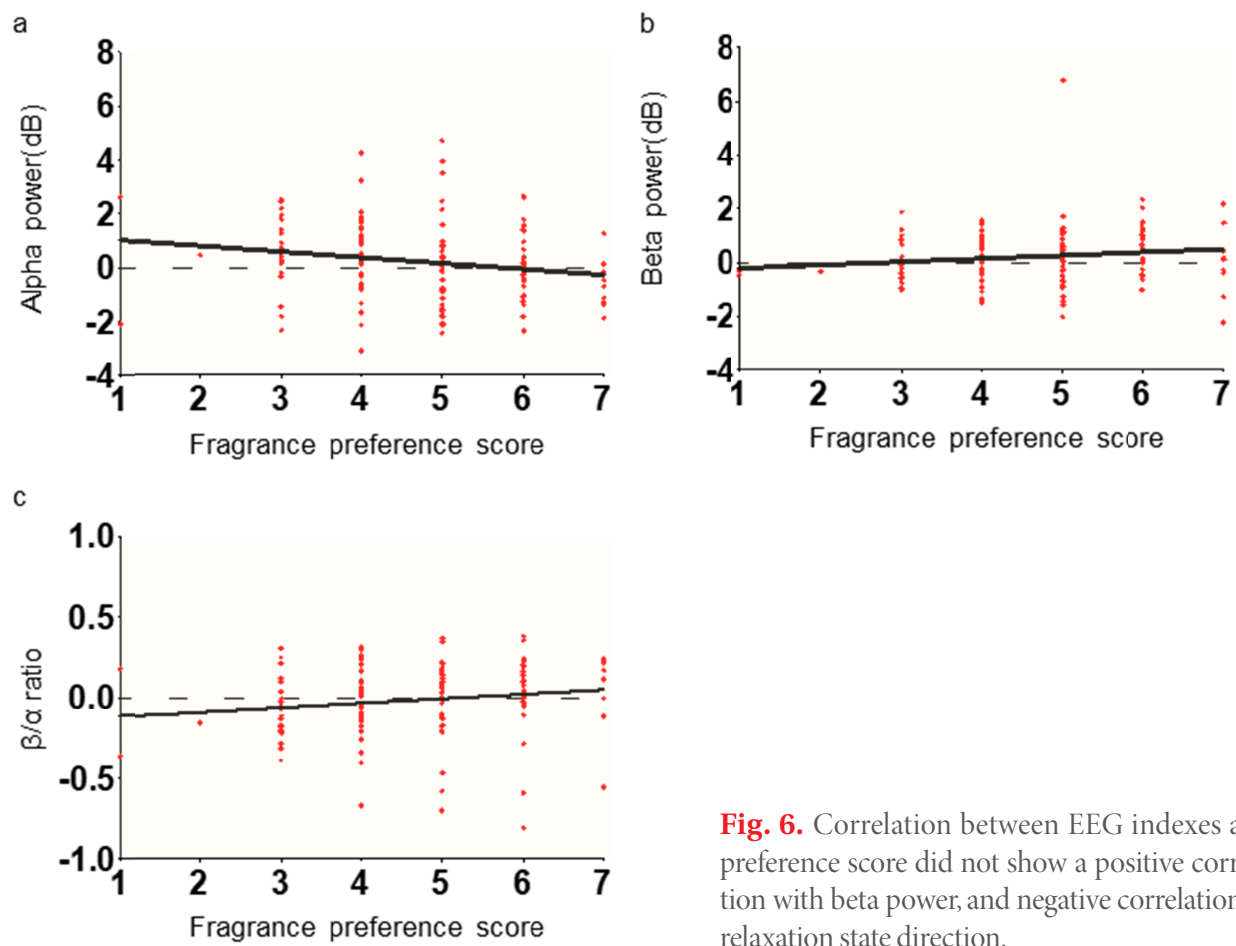

Fig. 6. Correlation between EEG indexes and fragrance preference score. Fragrance preference score did not show a positive correlation with alpha power, negative correlation with beta power, and negative correlation with $\beta / \alpha$ ratio, which is known as the EEG relaxation state direction.

However, under our experimental condition, we did not observe such changes in other frequency band powers (i.e., theta, beta, gamma) and frontal alpha asymmetry (FAA). According to a previous study, menopausal syndrome patients with depression showed significantly different EEG mapping with healthy women [9]. The patients characterized by decreased total power and attenuated alpha power. Furthermore, they showed the lower FAA in menopausal syndrome patients with depression than healthy women. Depressed patients showed lower alpha power in the right than the left frontal lobe. We also expected that total power and FAA are decreased in women with severe menopausal symptoms, however, there was no significant difference. The reason for the different results is probably we recruited participants who had no diagnosis of neurological disorders and used NAPS for the stimuli for our experimental design. The FAA pattern reported that decreased only at CES-D greater than 16 [52], which is a cut-off for depression diagnosis [53] (depression is diagnosed at CES-D score $>16$ ). Since we recruited healthy mid-life women (not depressed patients), the FAA pattern seems different. In addition, a previous study measured EEG during lying relaxed with closed eyes. In our study, to set up an event-related and controlled brain state among participants, we presented negative affective pictures during EEG recording. This set up allowed us to find psychological symptomspecific modulation of alpha power and $\beta / \alpha$ ratio in the response of stressful situations in the mid-life women.
Our novel finding is fragrances reduced the difference of brain response to stressful stimuli between participants with severe and light psychological symptoms. Participants answered that most of the presented fragrances have relaxed effects after stressful stimuli (Fig. 3). However, fragrance preference survey ratings were not reflected in EEG signals (Fig. 6). Instead of a hedonic evaluation of fragrance, alpha power, and $\beta / \alpha$ ratio were changed by each presented fragrance depending on the severity of anxiety or depressive symptoms (Fig. 4). In no-odor condition, participants with severe psychological symptoms seemed to have lower stress coping ability in the brain than participants with light psychological symptoms (Fig. 2, Table 1). The reason why we interpret this result is that their linear regression line showed a larger alpha and $\beta / \alpha$ ratio difference from the baseline than participants with light psychological symptoms. To alleviate the psychological menopausal symptoms, stress coping ability is important because menopausal symptoms could be severe, depending on the stressful situation coping skills [54]. For the stress coping and emotion regulation in the brain, oxytocin receptor-mediated actions were reported [5557]. And estrogen is the key factor for the oxytocin receptor binding [58]. During perimenopause, estradiol fluctuation occurs frequently, and it is reported that enhancing the emotional sensitivity to stress [59]. For further study, it would be interesting to reveal whether inhalation of fragrance has the role of oxytocin receptor binding in the perimenopausal women. 
We observed that lavender and O29 showed better efficacy in the process of returning brain signals similarly with baseline brain signals (Fig. 4, Fig. 5). Notably, we confirmed that lavender is an effective fragrance in case of anxiety symptoms in mid-life women using our method. Lavender has already been well characterized in terms of its anxiolytic, sedative, anti-depressive, and calming properties [34-36]. Moreover, the anxiolytic effect of lavender is reportedly accompanied by changes in the EEG pattern in humans [43]. Lavender itself might act as an efficient modulator of anxiety states in the mid-life women's brain compared to other fragrances. In the case of depressive symptoms, O29 had distinguished efficacy, and olibanum also showed modulating effects in our study. Previous studies had been reported components of olibanum, the Incensole acetate, showed reducing depressive symptoms. This component is known to reduce depressive-like behavior and is used in ceremonies to evoke the feeling of exaltation [44-46]. Our finding indicated that the chemical properties of fragrance seemed to reflect with the brain signal. Interestingly, the blended oils (O29, and P12) showed a more significant effect than olibanum, and palmarosa, probably due to the synergy effect of fragrances, and this needs to be verified in further study.

In this study, we revealed the fragrance-EEG index-menopausal symptom severity links. Our finding shows that EEG indexes, which are related to relaxing effects, are regulated by fragrance, and this fragrances effect varies depending on the severity of menopausal symptoms. Our approach may facilitate the development of a new repertoire of fragrances to establish a database of effective fragrances because our experimental scheme provides standard conditions to measure affective brain responses. Furthermore, using this database, it may be possible to recommend a series of fragrances depending on an individual's menopausal symptoms by using simple measurements. It might also be possible to predict brain responses or menopausal symptom severity using our method. In addition, it could be used to confirm the mood regulation effects of other sensory systems (e.g. music, scenery) in midlife women. To sum up this, fragrances could influence a woman's response to a stressful situation in daily life, and the impact on her social and personal life. Thereby our study might help to understand how mid-life women respond to various stimuli and could be widely used for the well-being of the society and of mid-life crisis sufferers.

\section{ACKNOWLEDGEMENTS}

This research was supported by the Convergent Technology R\&D Program for Human Augmentation funded through the National Research Foundation of Korea (NRF) by the Ministry of
Science and ICT (2019M3C1B8090845), Basic Science Research Program funded through the NRF by the Ministry of Education (2020R1A6A1A03040516), and AMOREPACIFIC R\&D Unit (RI5A000002).

\section{REFERENCES}

1. Brinton RD, Yao J, Yin F, Mack WJ, Cadenas E (2015) Perimenopause as a neurological transition state. Nat Rev Endocrinol 11:393-405.

2. McKinlay SM, Brambilla DJ, Posner JG (1992) The normal menopause transition. Maturitas 14:103-115.

3. Daly E, Gray A, Barlow D, McPherson K, Roche M, Vessey M (1993) Measuring the impact of menopausal symptoms on quality of life. BMJ 307:836-840.

4. Cohen LS, Soares CN, Vitonis AF, Otto MW, Harlow BL (2006) Risk for new onset of depression during the menopausal transition: the Harvard study of moods and cycles. Arch Gen Psychiatry 63:385-390.

5. Bromberger JT, Matthews KA, Schott LL, Brockwell S, Avis NE, Kravitz HM, Everson-Rose SA, Gold EB, Sowers M, Randolph JF Jr (2007) Depressive symptoms during the menopausal transition: the study of Women's Health Across the Nation (SWAN). J Affect Disord 103:267-272.

6. Campbell KE, Dennerstein L, Finch S, Szoeke CE (2017) Impact of menopausal status on negative mood and depressive symptoms in a longitudinal sample spanning 20 years. Menopause 24:490-496.

7. Frey BN, Hall GB, Attard S, Yucel K, Skelin I, Steiner M, Soares $\mathrm{CN}$ (2010) Shift in the brain network of emotional regulation in midlife women: is the menopausal transition the turning point? Menopause 17:840-845.

8. Etkin A, Egner T, Peraza DM, Kandel ER, Hirsch J (2006) Resolving emotional conflict: a role for the rostral anterior cingulate cortex in modulating activity in the amygdala. Neuron 51:871-882.

9. Saletu B, Brandstätter N, Metka M, Stamenkovic M, Anderer P, Semlitsch HV, Heytmanek G, Huber J, Grünberger J, Linzmayer L, Kurz C, Decker K, Binder G, Knogler W, Koll B (1996) Hormonal, syndromal and EEG mapping studies in menopausal syndrome patients with and without depression as compared with controls. Maturitas 23:91-105.

10. McCarrey AC, Resnick SM (2015) Postmenopausal hormone therapy and cognition. Horm Behav 74:167-172.

11. Pruis TA, Roalf DR, Janowsky JS (2009) Hormone therapy does not modify emotion-induced brain activity in older women. Horm Behav 56:539-547. 
12. Rossouw JE, Anderson GL, Prentice RL, LaCroix AZ, Kooperberg C, Stefanick ML, Jackson RD, Beresford SA, Howard BV, Johnson KC, Kotchen JM, Ockene J; Writing Group for the Women's Health Initiative Investigators (2002) Risks and benefits of estrogen plus progestin in healthy postmenopausal women: principal results from the Women's Health Initiative randomized controlled trial. JAMA 288:321-333.

13. Tao M, Teng Y, Shao H, Wu P, Mills EJ (2011) Knowledge, perceptions and information about hormone therapy (HT) among menopausal women: a systematic review and metasynthesis. PLoS One 6:e24661.

14. de Araujo IE, Rolls ET, Velazco MI, Margot C, Cayeux I (2005) Cognitive modulation of olfactory processing. Neuron 46:671-679.

15. Ferdenzi C, Delplanque S, Barbosa P, Court K, Guinard JX, Guo T, Roberts SC, Schirmer A, Porcherot C, Cayeux I, Sander D, Grandjean D (2013) Affective semantic space of scents. Towards a universal scale to measure self-reported odorrelated feelings. Food Qual Prefer 30:128-138.

16. Ferdenzi C, Roberts SC, Schirmer A, Delplanque S, Cekic S, Porcherot C, Cayeux I, Sander D, Grandjean D (2013) Variability of affective responses to odors: culture, gender, and olfactory knowledge. Chem Senses 38:175-186.

17. Rétiveau AN, Chambers E IV, Milliken GA (2004) Common and specific effects of fine fragrances on the mood of women. J Sens Stud 19:373-394.

18. Schiffman SS, Miller EA, Suggs MS, Graham BG (1995) The effect of environmental odors emanating from commercial swine operations on the mood of nearby residents. Brain Res Bull 37:369-375.

19. Schiffman SS, Sattely-Miller EA, Suggs MS, Graham BG (1995) The effect of pleasant odors and hormone status on mood of women at midlife. Brain Res Bull 36:19-29.

20. Warrenburg S (2005) Effects of fragrance on emotions: moods and physiology. Chem Senses 30 Suppl 1:i248-i249.

21. Lorig TS, Schwartz GE (1988) Brain and odor: I. alteration of human EEG by odor administration. Psychobiology 16:281284.

22. Yeshurun Y, Sobel N (2010) An odor is not worth a thousand words: from multidimensional odors to unidimensional odor objects. Annu Rev Psychol 61:219-241, C1-C5.

23. Krusemark EA, Novak LR, Gitelman DR, Li W (2013) When the sense of smell meets emotion: anxiety-state-dependent olfactory processing and neural circuitry adaptation. J Neurosci 33:15324-15332.

24. Novak LR, Gitelman DR, Schuyler B, Li W (2015) Olfactoryvisual integration facilitates perception of subthreshold nega- tive emotion. Neuropsychologia 77:288-297.

25. Gottfried JA (2010) Central mechanisms of odour object perception. Nat Rev Neurosci 11:628-641.

26. Carmichael ST, Clugnet MC, Price JL (1994) Central olfactory connections in the macaque monkey. J Comp Neurol 346:403-434.

27. Darsareh F, Taavoni S, Joolaee S, Haghani H (2012) Effect of aromatherapy massage on menopausal symptoms: a randomized placebo-controlled clinical trial. Menopause 19:995-999.

28. Taavoni S, Darsareh F, Joolaee S, Haghani H (2013) The effect of aromatherapy massage on the psychological symptoms of postmenopausal Iranian women. Complement Ther Med 21:158-163.

29. Cha JH, Kim MJ, Kim HS, Kim YI (2010) Effects of aromatherapy in blending oil of basil, lavender, rosemary, and rose on headache, anxiety and serum cortisol level in the middleaged women. J Korean Biol Nurs Sci 12:133-139.

30. Hur MH, Yang YS, Lee MS (2008) Aromatherapy massage affects menopausal symptoms in Korean climacteric women: a pilot-controlled clinical trial. Evid Based Complement Alternat Med 5:325-328.

31. Nie D, Wang XW, Shi LC, Lu BL. EEG-based emotion recognition during watching movies. Paper presented at: 2011 5th International IEEE/EMBS Conference on Neural Engineering; 2011 Apr 27-May 1; Cancun, Mexico.

32. Bos DO (2006) EEG-based emotion recognition. The influence of visual and auditory stimuli. CiteSeer ${ }^{\mathrm{X}}$ 56:1-17.

33. Marchewka A, Zurawski Ł, Jednoróg K, Grabowska A (2014) The Nencki Affective Picture System (NAPS): introduction to a novel, standardized, wide-range, high-quality, realistic picture database. Behav Res Methods 46:596-610.

34. Koulivand PH, Khaleghi Ghadiri M, Gorji A (2013) Lavender and the nervous system. Evid Based Complement Alternat Med 2013:681304.

35. Moss M, Cook J, Wesnes K, Duckett P (2003) Aromas of rosemary and lavender essential oils differentially affect cognition and mood in healthy adults. Int J Neurosci 113:15-38.

36. Motomura N, Sakurai A, Yotsuya Y (2001) Reduction of mental stress with lavender odorant. Percept Mot Skills 93:713718.

37. Alder E (1998) The Blatt-Kupperman menopausal index: a critique. Maturitas 29:19-24.

38. Rumeau C, Nguyen DT, Jankowski R (2016) How to assess olfactory performance with the Sniffin' Sticks test $\left({ }^{\circledR}\right)$. Eur Ann Otorhinolaryngol Head Neck Dis 133:203-206.

39. Greene JG (2008) Constructing a standard climacteric scale. Maturitas 61:78-84. 
40. Braithwaite JJ, Watson DG, Jones R, Rowe M (2013) A guide for analysing electrodermal activity (EDA) \& skin conductance responses (SCRs) for psychological experiments [Internet]. University of Birmingham, Birmingham. Available from: https://www.birmingham.ac.uk/Documents/college-les/ psych/saal/guide-electrodermal-activity.pdf.

41. De Raedt R, Franck E, Fannes K, Verstraeten E (2008) Is the relationship between frontal EEG alpha asymmetry and depression mediated by implicit or explicit self-esteem? Biol Psychol 77:89-92.

42. Delorme A, Makeig S (2004) EEGLAB: an open source toolbox for analysis of single-trial EEG dynamics including independent component analysis. J Neurosci Methods 134:9-21.

43. Field T, Diego M, Hernandez-Reif M, Cisneros W, Feijo L, Vera Y, Gil K, Grina D, Claire He Q (2005) Lavender fragrance cleansing gel effects on relaxation. Int J Neurosci 115:207-222.

44. Moussaieff A, Mechoulam R (2009) Boswellia resin: from religious ceremonies to medical uses; a review of in-vitro, invivo and clinical trials. J Pharm Pharmacol 61:1281-1293.

45. Moussaieff A, Gross M, Nesher E, Tikhonov T, Yadid G, Pinhasov A (2012) Incensole acetate reduces depressive-like behavior and modulates hippocampal BDNF and CRF expression of submissive animals. J Psychopharmacol 26:15841593.

46. Moussaieff A, Rimmerman N, Bregman T, Straiker A, Felder CC, Shoham S, Kashman Y, Huang SM, Lee H, Shohami E, Mackie K, Caterina MJ, Walker JM, Fride E, Mechoulam R (2008) Incensole acetate, an incense component, elicits psychoactivity by activating TRPV3 channels in the brain. FASEB J 22:3024-3034.

47. Babakhanian M, Ghazanfarpour M, Kargarfard L, Roozbeh N, Darvish L, Khadivzadeh T, Dizavandi FR (2018) Effect of aromatherapy on the treatment of psychological symptoms in postmenopausal and elderly women: a systematic review and meta-analysis. J Menopausal Med 24:127-132.

48. Kim S, Song JA, Kim ME, Hur MH (2016) Effects of aromatherapy on menopausal symptoms, perceived stress and depression in middle-aged women: a systematic review. J Korean Acad Nurs 46:619-629.
49. Koelstra S, Muhl C, Soleymani M, Lee JS, Yazdani A, Ebrahimi T, Pun T, Nijholt A, Patras I (2011) DEAP: a database for emotion analysis; using physiological signals. IEEE Trans Affect Comput 3:18-31.

50. Crabbe JB, Dishman RK (2004) Brain electrocortical activity during and after exercise: a quantitative synthesis. Psychophysiology 41:563-574.

51. Ramirez R, Vamvakousis Z (2012) Detecting emotion from EEG signals using the emotive Epoc device. In: Lecture notes in computer science (Bertino E, Gao W, Steffen B, Woeginger G, Yung M, eds), pp 175-184. Springer Science+Business Media, Berlin.

52. Diego MA, Field T, Hernandez-Reif M (2001) CES-D depression scores are correlated with frontal EEG alpha asymmetry. Depress Anxiety 13:32-37.

53. Vilagut G, Forero CG, Barbaglia G, Alonso J (2016) Screening for depression in the general population with the center for epidemiologic studies depression (CES-D): a systematic review with meta-analysis. PLoS One 11:e0155431.

54. Ballinger SE (1985) Psychosocial stress and symptoms of menopause: a comparative study of menopause clinic patients and non-patients. Maturitas 7:315-327.

55. Neumann ID (2008) Brain oxytocin: a key regulator of emotional and social behaviours in both females and males. J Neuroendocrinol 20:858-865.

56. Amico JA, Mantella RC, Vollmer RR, Li X (2004) Anxiety and stress responses in female oxytocin deficient mice. J Neuroendocrinol 16:319-324.

57. Neumann ID (2002) Involvement of the brain oxytocin system in stress coping: interactions with the hypothalamopituitary-adrenal axis. Prog Brain Res 139:147-162.

58. Young LJ, Wang Z, Donaldson R, Rissman EF (1998) Estrogen receptor alpha is essential for induction of oxytocin receptor by estrogen. Neuroreport 9:933-936.

59. Gordon JL, Rubinow DR, Eisenlohr-Moul TA, Leserman J, Girdler SS (2016) Estradiol variability, stressful life events, and the emergence of depressive symptomatology during the menopausal transition. Menopause 23:257-266. 